Article

\title{
Model for the Evaluation of an Angular Slot's Coupling Impedance
}

\author{
Dario Assante ${ }^{1, *(1)}$ and Luigi Verolino ${ }^{2}$ (D) \\ 1 Facoltà di Ingegneria, Università Telematica Internazionale Uninettuno, 00186 Roma, Italy \\ 2 Dipartimento di Ingegneria Elettrica e delle Tecnologie dell'Informazione, Università degli Studi di Napoli \\ Federico II, 80138 Napoli, Italy; verolino@unina.it \\ * Correspondence: d.assante@uninettunouniversity.net
}

Received: 25 April 2019; Accepted: 11 May 2019; Published: 22 May 2019

\begin{abstract}
In high energy particle accelerators, a careful modeling of the electromagnetic interaction between the particle beam and the structure is essential to ensure the performance of the experiments. Particular interest arises in the presence of angular discontinuities of the structure, due to the asymmetrical behavior. In this case, semi-analytical models allow one to reduce the computational effort and to better understand the physics of the phenomena, with respect to purely numerical models. In the paper, a model for analyzing the electromagnetic interaction between a traveling charge particle and a perfectly conducting angular slot of a negligible thickness is discussed. The particle travels at a constant velocity along a straight line parallel to the axis of symmetry of the strip. The longitudinal and transverse coupling impedances are therefore evaluated for a wide range of parameters.
\end{abstract}

Keywords: particle accelerator; coupling impedance; dual integral equations

\section{Introduction}

Recent discoveries in high-energy particle accelerators are connected to the possibility to reach higher level of energies in the experiments [1]. One of the main limitations to the involved energy, that is to say to the current of the beam, is the instability of the particle due to the electromagnetic interaction with the surrounding structures [2]. The synthetic design parameter commonly adopted in literature to describe the electromagnetic interaction between a traveling particle and a structure is the coupling impedance [3-5]. This parameter is proportional to the energy lost by the traveling charge due to the interaction with the scattered fields produced by the surrounding structures. Equivalently, it is proportional to the energy that has to be spent to keep its speed constant, neglecting the slowing effect of the surrounding structures. For structures invariant along the charge traveling direction, a per-unit-length coupling impedance has to be introduced [4], whose longitudinal and transverse components can be defined as

$$
Z_{\|}(r, \varphi, k)=-\frac{1}{q} \frac{1}{L} \int_{-L / 2}^{L / 2} E_{z}(r, \varphi, z, \omega) e^{j k z / \beta} d z \quad Z_{\perp}(r, \varphi, k)=\frac{1}{k} \nabla_{\perp} Z_{\|}(r, \varphi, k),
$$

where $L$ is an unitary length, $E_{z}(r, \varphi, z, \omega)$ the x-component of the electric field in the frequency domain, $k$ the wavenumber, and the charge $q$ is moving at constant velocity $v=\beta c$ along the $z$ axis. The second equation in (1) is known as the Panofski-Wenzel theorem [6].

The research of new shapes of cavities with proper coupling impedances is actually of high interest for the design of even more efficient particle accelerators [7-9]. Nowadays, powerful tools allow 
performing the electromagnetic numerical analysis of complex structures [10]. However, analytical or semi-analytical solutions still play a valuable role in this field, enabling to better understand the physics of some phenomena. Modal analysis is often adopted for close structures [11,12], diffractive methods for high-frequency solutions, and integral formulations for open geometries or in the presence of edges $[13,14]$.

Most of the studies related to the coupling impedance consider, in a cylindrical reference system, axially symmetric geometries. This choice is both because they represent most of the structures of interest and because the symmetry allows finding the solution with less effort or even in a semi-analytical form. In this paper, we want to analyze the interaction of a particle with a axially asymmetric structure, in particular an angular slot, as shown in Figure 1. This configuration is representative of particle accelerator components that break the axial symmetry. The proposed method is quite general and can be adopted for a wide class of scattering and diffraction problems [15-20]. It can be easily generalized and adopted to analyze similar geometries.
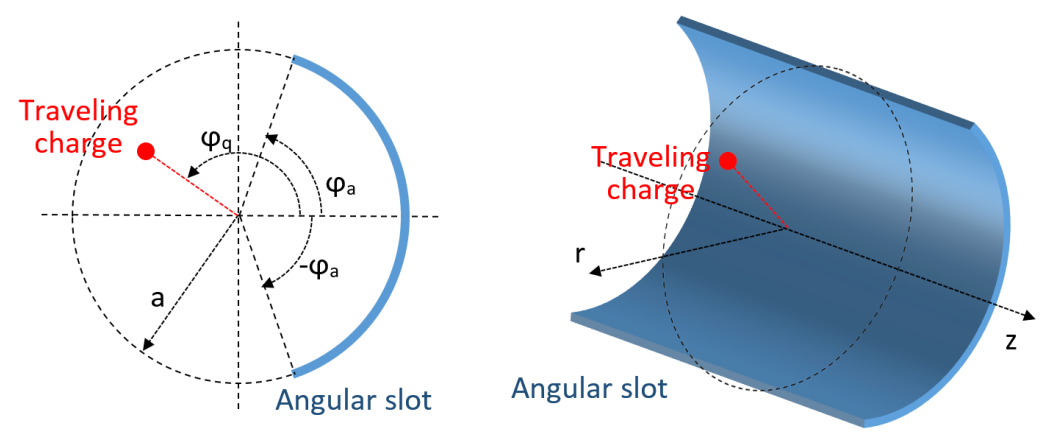

Figure 1. Geometry of the problem.

The problem is formulated in the particle frame at first. Assuming the geometry invariant along the traveling direction, in the particle frame a stationary model is adoptable, this simplifies the formulation and the solution. In order to evaluate the coupling impedance, the electromagnetic quantities are then obtained in the slot frame by means of Lorentz transforms.

The angular slot is assumed to be perfectly conductive; this is a common choice in the literature. Although the finite conductivity of the strip can be taken into account with some complications, neglecting it does not have a concrete effect on the validity of the analysis. The validity of such a choice is discussed more in detail in the last section of the paper.

The primed notation is adopted to identify the quantities in the particle frame, the unprimed notation in the slot frame.

The paper is composed of 6 sections: after this introduction, in the next section the problem is formulated in the particle frame and a methodology for computing the unknown current density is presented. In the following sections, the electromagnetic fields are evaluated in the particle and in the structure frame and then the coupling impedance is estimated. Then, some numerical results are presented. Finally, the conclusions are discussed.

\section{Formulation of the Problem in the Particle Frame}

In this section let us consider the geometry shown in Figure 1: a perfectly conducting angular slot $\mathbb{S}=\left\{r=a,|\varphi| \leq \varphi_{a}, z\right\}$ at distance $a$ from the axis and covering an angular sector of $2 \varphi_{a}$. A travelling charge $q$ moves parallel to the slots's axis, placed at $\left(r_{q}, \varphi_{q}\right)$, at constant speed $v=\beta c, c$ being the speed of light in free space.

The problem is formulated in term of integral equations and its solution is reduced to the resolution of a linear system.

The electromagnetic interaction between the particle and the structure can be easily formulated and solved in the particle frame, being an electrostatic model adequate for such a problem. Once the 
electromagnetic quantities are computed, their values in the slot frame can be obtained by means of Lorentz transforms.

The electrostatic potential produced by the charge is

$$
V_{q}^{\prime}=\frac{q}{4 \pi \varepsilon_{0} \sqrt{r^{\prime 2}+r_{q}^{2}-2 r^{\prime} r_{q} \cos \left(\varphi^{\prime}-\varphi_{q}\right)+z^{\prime 2}}}
$$

while the potential produced by the induced charge density $\sigma^{\prime}\left(\varphi^{\prime}, z^{\prime}\right)$ on the slot can be expressed as

$$
V^{\prime}=\frac{a}{4 \pi \varepsilon_{0}} \int_{\mathbb{S}} \frac{\sigma^{\prime}\left(\varphi_{0}, z_{0}\right) d \varphi_{0} d z_{0}}{\sqrt{r^{\prime 2}+a^{2}-2 r^{\prime} a \cos \left(\varphi^{\prime}-\varphi_{0}\right)+\left(z^{\prime}-z_{0}\right)^{2}}} .
$$

Being a perfectly conducting slot, the boundary condition to be verified is that the tangential components of the electric field vanishes on the slot. This corresponds to impose that

$$
V^{\prime}\left(r^{\prime}=a, \varphi^{\prime}, z\right)+V_{q}^{\prime}\left(r^{\prime}=a, \varphi^{\prime}, z\right)=0
$$

for every $\left(\varphi^{\prime}, z^{\prime}\right) \in \mathbb{S}$.

Considering Equations (2) and (3), the boundary condition leads to

$$
\int_{\mathbb{S}} \frac{\sigma^{\prime}\left(\varphi_{0}, z_{0}\right) d \varphi_{0} d x_{0}}{\sqrt{2 a^{2}-2 a^{2} \cos \left(\varphi^{\prime}-\varphi_{0}\right)+\left(z^{\prime}-z_{0}\right)^{2}}}=-\frac{q / a}{\sqrt{a^{2}+r_{q}^{2}-2 a r_{q} \cos \left(\varphi^{\prime}-\varphi_{q}\right)+z^{\prime 2}}}
$$

From this equation, it is worth noting to observe that there is complete induction on the slot for such a kind of geometry. In fact, by multiplying Equation (5) for the denominator of its second member and performing a limit for $z$ going toward $+\infty$, it is possible to obtain that

$$
\int_{\mathbb{S}} \sigma^{\prime}\left(\varphi_{0}, z_{0}\right) a d \varphi_{0} d z_{0}=-q
$$

This result will be usefully employed later on in the computation of the coupling impedance. In order to solve the problem it is necessary to recall a relevant integral ([21] R6.616.4)

$$
\frac{\pi}{\sqrt{D^{2}+Z^{2}}}=\int_{-\infty}^{+\infty} K_{0}(D w) e^{-j w Z} d w
$$

and its derived form

$$
\frac{\pi Z}{\left(D^{2}+Z^{2}\right)^{3 / 2}}=j \int_{-\infty}^{+\infty} w K_{0}(D w) e^{-j w Z} d w .
$$

Then it is useful to introduce a spatial Fourier transform along the $z$ axis, namely

$$
\tilde{\sigma}^{\prime}(\varphi, w)=\frac{1}{2 \pi} \int_{-\infty}^{+\infty} \sigma^{\prime}(\varphi, z) e^{j w z} d z
$$

By using Equation (7) on the integral Equation (5) and applying the inverse Fourier transform to both members, with some manipulations it becomes 


$$
\begin{aligned}
& \int_{-\varphi_{a}}^{\varphi_{a}} \tilde{\sigma}^{\prime}\left(\varphi_{0}, w\right) K_{0}\left(\sqrt{2} a w \sqrt{1-\cos \left(\varphi^{\prime}-\varphi_{0}\right)}\right) d \varphi_{0}= \\
& =-\frac{q}{2 \pi a} K_{0}\left(a w \sqrt{1+\left(r_{q} / a\right)^{2}-2\left(r_{q} / a\right) \cos \left(\varphi^{\prime}-\varphi_{q}\right)}\right) .
\end{aligned}
$$

Equation (10) has to be verified on $\mathbb{S}$, whereas the induced charge density vanishes outside the angular slot, namely

$$
\int_{-\infty}^{+\infty} \tilde{\sigma}^{\prime}\left(\varphi_{0}, w\right) e^{-j w z} d w=0
$$

Equations (10) and (11) constitute a dual system of integral equation with respect to the induced current density. An efficient solution of such a problem can be obtained by representing the unknown in terms of Neuman series [22]:

$$
\sigma^{\prime}\left(\varphi^{\prime}, z^{\prime}\right)= \begin{cases}-\frac{q}{2 \pi a \varphi_{a}} \sum_{n=0}^{\infty} \sigma_{n}\left(z^{\prime}\right) \frac{T_{n}\left(\varphi^{\prime} / \varphi_{a}\right)}{\sqrt{1-\left(\varphi^{\prime} / \varphi_{a}\right)^{2}}}, & |\varphi| \leq \varphi_{a} \\ 0, & |\varphi|>\varphi_{a}\end{cases}
$$

where $T_{n}(\cdot)$ is the Chebychev polynomial of order $n[23,24]$. Such polynomials exhibit several relevant properties $[25,26]$ that can be adopted for the solution of some classes of electromagnetic problems. According to Equation (9), then the induced current density in the transformed domain is

$$
\tilde{\sigma}^{\prime}\left(\varphi^{\prime}, w\right)=-\frac{q}{2 \pi a \varphi_{a}} \sum_{n=0}^{\infty} \tilde{\sigma}_{n}(w) \frac{T_{n}\left(\varphi^{\prime} / \varphi_{a}\right)}{\sqrt{1-\left(\varphi^{\prime} / \varphi_{a}\right)^{2}}} .
$$

With such a normalization, dimensionless expansion coefficients are obtained in the transformed domain.

The chosen representation is a form of the more generic Neuman series, particularized for this problem [27]. The chosen representation automatically matches the right edge behavior, this regularizes the method and reduces the number of required coefficients.

In addition, the chosen current density representation already satisfies Equation (11). So, by substituting it into the remaining Equation (10), it is found that

$$
\begin{aligned}
\sum_{n=0}^{\infty} \tilde{\sigma}_{n}(w) \int_{-\varphi_{a}}^{\varphi_{a}} \frac{T_{n}\left(\varphi^{\prime} / \varphi_{a}\right)}{\sqrt{1-\left(\varphi^{\prime} / \varphi_{a}\right)^{2}}} K_{0}\left(\sqrt{2} a w \sqrt{1-\cos \left(\varphi^{\prime}-\varphi_{0}\right)}\right) d \varphi_{0}= \\
=\varphi_{a} K_{0}\left(a w \sqrt{1+\left(r_{q} / a\right)^{2}-2\left(r_{q} / a\right) \cos \left(\varphi^{\prime}-\varphi_{q}\right)}\right) .
\end{aligned}
$$

This equation has to be verified for every $\left|\varphi^{\prime}\right| \leq \varphi_{a}$. In order to impose this condition, Equation (14) is projected on the same basis functions adopted for the representation of the current density (Galerkin scheme). This converts Equation (14) in the linear system

$$
A \tilde{\sigma}=b,
$$

where $\tilde{\sigma}$ is the vector of the unknowns $\tilde{\sigma}_{n}, A$ is a symmetric matrix whose coefficients, obtained with some trivial manipulations and changes of variable as

$$
A_{n m}=\int_{0}^{\pi} \int_{0}^{\pi} K_{0}\left(2 a w\left|\sin \left(\frac{\varphi_{a}}{2}\left(\cos \psi_{0}-\cos \psi^{\prime}\right)\right)\right|\right) \cos \left(m \psi_{0}\right) \cos \left(n \psi^{\prime}\right) d \psi_{0} d \psi^{\prime}
$$


and $\boldsymbol{b}$ the known term vector defined as

$$
b_{m}=\int_{0}^{\pi} K_{0}\left(a w \sqrt{1+\left(r_{q} / a\right)^{2}-2\left(r_{q} / a\right) \cos \left(\varphi_{a} \cos \psi^{\prime}-\varphi_{q}\right)}\right) \cos \left(m \psi^{\prime}\right) d \psi^{\prime} .
$$

It is worth noting that for $r_{q}=0$, that is to say when the particle is in the axis of the slot, all the coefficients $b_{m}$ are zero but the first one, that is $b_{0}=\pi K_{0}(a w)$.

\section{Electromagnetic Fields in the Slot Frame}

In order to complete the problem formulation, it is proper to express the electromagnetic fields in the slot frame, too. This can be realized by applying the Lorentz transforms to the fields computed in the previous section in particle frame.

Let us consider at first the $z$ component of the electric field. The contribution provided by the traveling charge in the frequency domain is well known and is

$$
E_{z, q}=\frac{j q \kappa \zeta_{0}}{2 \pi \beta \gamma} e^{-j z k / \beta} K_{0}\left(\kappa \sqrt{r^{2}+r_{q}^{2}-2 r r_{q} \cos (\varphi)}\right),
$$

where $\gamma=1 / \sqrt{1-\beta^{2}}$ is the Lorentz factor, $\kappa=k /(\beta \gamma)$, and $\zeta_{0}=\sqrt{\mu_{0} / \varepsilon_{0}}$ is the characteristic impedance of free space.

The contribution produced by the induced current density on the slot can be obtained with some manipulations as function of the representation coefficients $\sigma_{n}$.

In the particle frame, starting from Equation (3) it is possible to obtain

$$
e_{z}^{\prime}\left(r^{\prime}, \varphi^{\prime}, z^{\prime}\right)=\frac{a}{4 \pi \varepsilon_{0}} \int_{\mathbb{S}} \frac{\sigma^{\prime}\left(\varphi_{0}, z_{0}\right)\left(z^{\prime}-z_{0}\right) d \varphi_{0} d z_{0}}{\left[r^{\prime 2}+a^{2}-2 r^{\prime} a \cos \left(\varphi^{\prime}-\varphi_{0}\right)+\left(z^{\prime}-z_{0}\right)^{2}\right]^{3 / 2}} .
$$

Lorentz transforms are now applied to obtain the electric field in the slot frame. In this specific case they are

$$
e_{z}^{\prime}=e_{z}, \sigma^{\prime}=\sigma \gamma, r^{\prime}=r, \varphi^{\prime}=\varphi, z^{\prime}=\gamma(z-v t) .
$$

Applying these transforms to Equation (19), it is found that

$$
e_{z}(r, \varphi, z, t)=\frac{a \gamma}{4 \pi \varepsilon_{0}} \int_{\mathbb{S}} \frac{\sigma\left(\varphi_{0}, z_{0}\right)\left(\gamma(z-v t)-z_{0}\right) d \varphi_{0} d z_{0}}{\left[r^{2}+a^{2}-2 r a \cos \left(\varphi-\varphi_{0}\right)+\left(\gamma(z-v t)-z_{0}\right)^{2}\right]^{3 / 2}} .
$$

By means of Equation (8) and applying a spatial Fourier transform according to Equation (9), it is found that

$$
e_{z}(r, \varphi, z, t)=\frac{j a \gamma}{2 \pi \varepsilon_{0}} \int_{-\varphi_{a}}^{+\varphi_{a}} \int_{-\infty}^{+\infty} \tilde{\sigma}\left(\varphi_{0}, w\right) w e^{j w \gamma v t} K_{0}\left(w \sqrt{r^{2}+a^{2}-2 r a \cos \left(\varphi-\varphi_{0}\right)}\right) e^{-j w \gamma z} d \varphi_{0} d w .
$$

Finally, by performing a time Fourier transform and then the integral on $w$, the required field is finally found as

$$
E_{z}(r, \varphi, z, \omega)=\frac{j a k \zeta_{0}}{\beta^{2}} e^{-j z k / \beta} \int_{-\varphi_{a}}^{+\varphi_{a}} \tilde{\sigma}\left(\varphi_{0}, \kappa\right) K_{0}\left(\kappa \sqrt{r^{2}+a^{2}-2 r a \cos \left(\varphi-\varphi_{0}\right)}\right) d \varphi_{0}
$$

The last integral can be performed by substituting the representation of the current density (13), leading to the very simple expression of the $z$-component of the electric field in the slot frame: 


$$
E_{z}(r, \varphi, z, \omega)=-\frac{j q k \zeta_{0}}{2 \pi \varphi_{a} \beta^{2}} e^{-j z k / \beta} \sum_{n=0}^{\infty} \tilde{\sigma}_{n}(\kappa) b_{n}(r, \varphi, \kappa),
$$

where coefficients $b_{n}(r, \varphi, \kappa)$ have the same expression of Equation (29) but are computed as the generic point $(r, \varphi)$ and for $w=\kappa$.

With a similar procedure, all the other fields can be expressed. For instance, it is possible to easily find that the charge density induced on the slot is

$$
\sigma(\varphi, z, \omega)=-\frac{q}{a \varphi_{a} \gamma} \sum_{n=0}^{\infty} \sigma_{n}(\kappa) \frac{T_{n}\left(\varphi / \varphi_{a}\right)}{\sqrt{1-\left(\varphi / \varphi_{a}\right)^{2}}} .
$$

\section{Coupling Impedance}

In order to compute the coupling impedance, just the longitudinal component of the electric field produced by the induced currents is required, since the electric field produced by the traveling charge does not contribute to the coupling impedance.

So, given Equation (24), the per-unit-length longitudinal coupling impedance (1) can be easily computed in a generic point in the transverse plane as

$$
Z_{\|}(r, \varphi, k)=\frac{j k \zeta_{0}}{2 \pi \varphi_{a} \beta^{2}} \sum_{n=0}^{\infty} \tilde{\sigma}_{n}(\kappa) b_{n}(\kappa, r, \varphi) .
$$

It is worth noting that, since the matrix and the known term vector in Equation (15) are purely real, all the unknown terms $\sigma_{n}$ are real, too. So, the longitudinal coupling impedance is purely imaginary. This result is expected since there are not diffraction losses.

From Equation (26), by means of the definition (1) it is possible to find the expression of the transverse coupling impedance, that is

$$
Z_{\perp}(r, \varphi, k)=\frac{j k \zeta_{0}}{2 \pi \varphi_{a} \beta^{2}} \sum_{n=0}^{\infty} \tilde{\sigma}_{n}(\kappa)\left\{\frac{\partial b_{n}(\kappa, r, \varphi)}{\partial r} \hat{r}+\frac{1}{r} \frac{\partial b_{n}(\kappa, r, \varphi)}{\partial \varphi} \hat{\varphi}\right\}
$$

In order to compute the transverse coupling impedance in a practical way, it is worth recalling the addition theorem for the Hankel functions

$$
K_{0}(w R)=\sum_{p=-\infty}^{+\infty}(-1)^{p} I_{p}\left(\rho^{\prime} w\right) K_{p}(\rho w) e^{j p\left(\varphi-\varphi^{\prime}\right)},
$$

being $R=\sqrt{\rho^{\prime 2}+\rho^{2}-2 \rho^{\prime} \rho \cos \left(\phi^{\prime}-\phi\right)}$ and $\rho^{\prime} \leq \rho$.

With some manipulations, it can be used to analytically compute the integral in Equation (17), expressing the coefficients $b_{n}$ as series of products of Bessel functions, namely

$$
b_{m}\left(r_{q}, \varphi_{q}, w\right)=\pi \begin{cases}I_{0}\left(r_{q} w\right) K_{0}(a w)+2 \sum_{p=1}^{+\infty}(-1)^{p} I_{p}\left(r_{q} w\right) K_{p}(a w) J_{0}\left(p \varphi_{a}\right) \cos \left(p \varphi_{q}\right), & m=0, \\ 2 j^{m} \sum_{p=1}^{+\infty}(-1)^{p} I_{p}\left(r_{q} w\right) K_{p}(a w) J_{m}\left(p \varphi_{a}\right) \cos \left(p \varphi_{q}\right), & m \text { even } \\ 2 j^{m} \sum_{p=1}^{+\infty}(-1)^{p} I_{p}\left(r_{q} w\right) K_{p}(a w) J_{m}\left(p \varphi_{a}\right) \sin \left(p \varphi_{q}\right), & m \text { odd } .\end{cases}
$$

Then, the derivatives required in Equation (27) can be expressed as 


$$
\frac{\partial b_{n}}{\partial r}(r, \varphi, \kappa)=2 \pi j^{n} \begin{cases}\frac{1}{2} I_{1}(r \kappa) K_{0}(a \kappa)+ & \\ +\sum_{p=1}^{+\infty}(-1)^{p}\left[\frac{p}{r} I_{p}(r \kappa)+I_{p+1}(r \kappa)\right] K_{p}(a \kappa) J_{0}\left(p \varphi_{a}\right) \cos (p \varphi), & m=0 \\ \sum_{p=1}^{+\infty}(-1)^{p}\left[\frac{p}{r} I_{p}(r \kappa)+I_{p+1}(r \kappa)\right] K_{p}(a \kappa) J_{n}\left(p \varphi_{a}\right) \cos (p \varphi), & n \text { even } \\ \sum_{p=1}^{+\infty}(-1)^{p}\left[\frac{p}{r} I_{p}(r \kappa)+I_{p+1}(r \kappa)\right] K_{p}(a \kappa) J_{n}\left(p \varphi_{a}\right) \sin (p \varphi), & n \text { odd }\end{cases}
$$

and

$$
\frac{1}{r} \frac{\partial b_{n}}{\partial \varphi}(r, \varphi, \kappa)=\frac{2 \pi j^{n}}{r} \begin{cases}\sum_{p=1}^{+\infty}(-1)^{(p+1)} p I_{p}(r \kappa) K_{p}(a \kappa) I_{n}\left(p \varphi_{a}\right) \sin (p \varphi), & n \text { even }, \\ \sum_{p=1}^{+\infty}(-1)^{(p+1)} p I_{p}(r \kappa) K_{p}(a \kappa) I_{n}\left(p \varphi_{a}\right) \cos (p \varphi), & n \text { odd } .\end{cases}
$$

Among all the possible positions in the transverse plane, a relevant one is when the particle is in the center of the slot, namely $r=0$. In fact, in a particle accelerator, the particle is supposed to travel along the radial axis of the structure, or eventually in a position very close to it. In this case, as previously stated, all the coefficients $b_{n}(\kappa, 0)$ vanishes but the one for $n=0$.

So the longitudinal coupling impedance Equation (26) assumes the very simple expression

$$
Z_{\|}(r, \varphi, k)=\frac{j k \zeta_{0}}{2 \pi \varphi_{a} \beta^{2}} \tilde{\sigma}_{0}(\kappa) b_{0}(\kappa, 0) .
$$

Then, performing the limits for $r$ going to zero of Equations (30) and (31), the coefficients of Equation (27) reduces to

$$
\frac{\partial b_{n}}{\partial r}(r, \varphi, \kappa)=-\pi j^{n} K_{1}(a \kappa) J_{n}\left(\varphi_{a}\right) \begin{cases}\cos (\varphi), & n \text { even }, \\ \sin (\varphi), & n \text { odd. }\end{cases}
$$

and

$$
\frac{1}{r} \frac{\partial b_{n}}{\partial \varphi}(r, \varphi, \kappa)=\pi j^{n} K_{1}(a \kappa) J_{n}\left(\varphi_{a}\right) \begin{cases}\sin (\varphi), & n \text { even }, \\ \cos (\varphi), & n \text { odd } .\end{cases}
$$

So the transverse coupling impedance (27) can be easily expressed in Cartesian coordinates and becomes

$$
Z_{\perp}(r, \varphi, k)=-\frac{j k \zeta_{0}}{2 \varphi_{a} \beta^{2}} K_{1}(a \kappa) \sum_{n=0}^{\infty} j^{n} \tilde{\sigma}_{n}(\kappa) J_{n}\left(\varphi_{a}\right) \hat{x} .
$$

Such a result is coherent with the physics of the problem since, due to symmetry reasons, for such particle position it is expected that the transverse coupling impedance is along the bisector of the angular slot.

\section{Numerical Results}

Some numerical results are presented in this section, in order to discuss the efficiency of the proposed method. In all the simulations, the shape of the angular slot is $a=1 \mathrm{~cm}, \varphi_{a}=60^{\circ}$. A Simpson rule with an adaptive spacing is adopted to compute the matrix coefficients (16), while a Gaussian quadrature algorithm is used for the coefficients (17). Since the kernel of the terms in Equation (16) exhibits a logarithmic singularity and gives rise to computational problems, proper numerical manipulations have to be introduced to navigate the problem. The adopted solution is discussed in the Appendix A. At first, the behavior of the coefficients $\sigma_{n}$ is shown for different values of the frequency and of the distance between the particle and the structure. In Figure $2 a$ the absolute values of expansion coefficients are shown for different frequencies. The particle is in the center of the axis, as that is the most realistic case in practice. At lower frequencies, the coefficients' amplitudes quickly 
decrease, with few of them being enough to properly represent the current density: for $a \kappa=0.01$, the third coefficient is already four orders of magnitude lower than the first one. At higher frequencies, the amplitude of the coefficients decreases more slowly, so an higher number of coefficients is required, as expected.

In Figure $2 b$ the coefficients are shown in case of offset of the particle beam. As expected, the amplitude of the coefficients increases as the distance of the particle from the structure decreases. Additionally, while the odd coefficients vanish in case of centred particle, they grow proportionally to the particle offset.

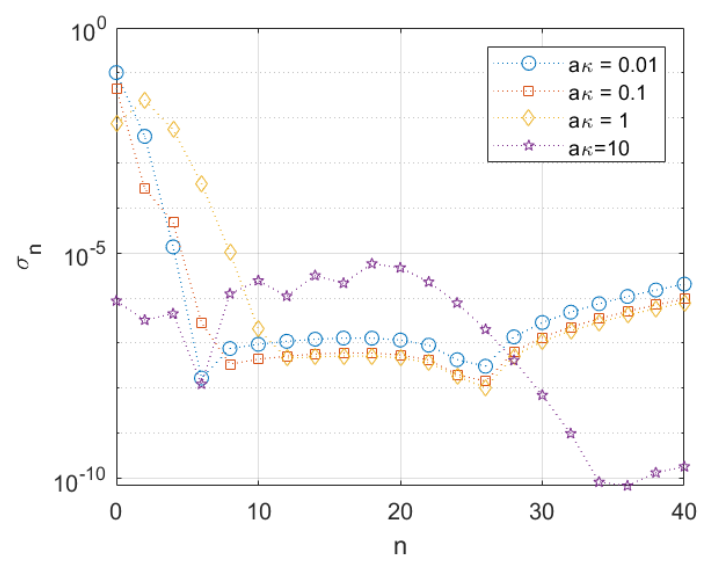

(a)

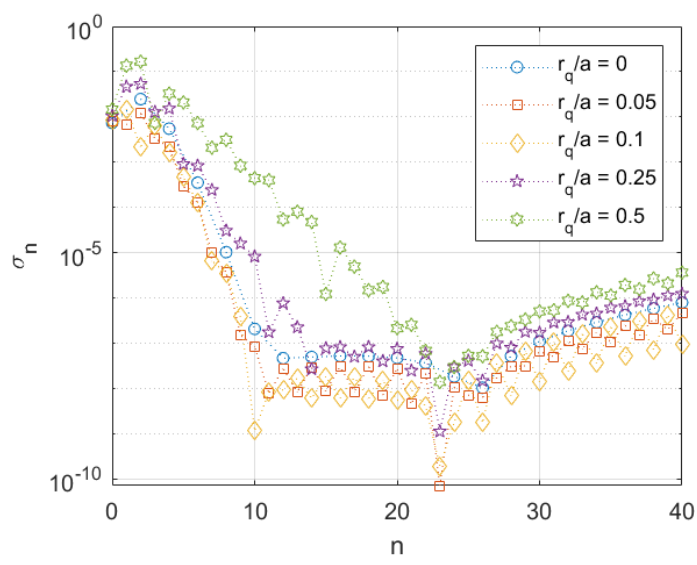

(b)

Figure 2. Absolute values of the expansion coefficients at $\beta \gamma=1$ for: (a) different frequencies $\left(r_{q}=0\right)$, (b) different offsets of the particle $\left(a \kappa=1, \varphi_{q}=30^{\circ}\right)$.

Then, in Figure 3 we show the behavior of the current density induced on the angular slot as in Equation (25), for different values of the frequency, normalized with respect to the charge. The adopted coefficients are the same as in Figure 2a. As expected, at low frequencies induced currents have a very flat behavior, just exhibiting a divergence at the boundaries. As the frequency grows, the behavior of the current density is more variable even in the middle of the angular slot. The results have been successfully validated with a finite element tool, providing a very good correspondence in the middle of the structure and being unable to reproduce the proper divergent boundary behavior, as expected.
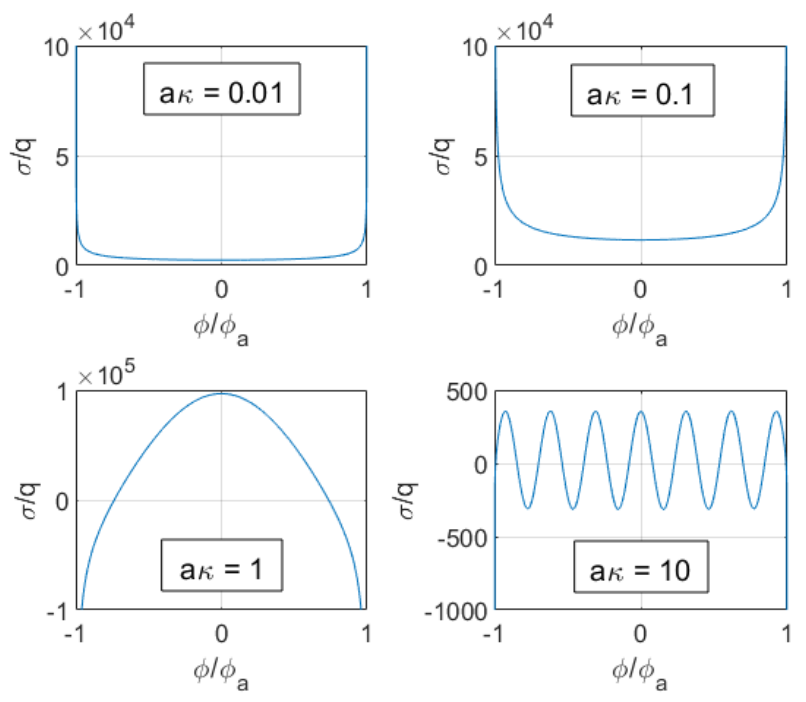

Figure 3. Behavior of the current density induced on the structure $\left(r_{q}=0, \beta \gamma=1\right)$. 
Finally, the coupling impedance is discussed. In Figure 4a, the longitudinal coupling impedance is shown, as a function of the frequency and of the particle speed. As already discussed, in practice it is a coupling reactance, the real part being null. The shape is slightly influenced by the particle speed. As expected, the coupling impedance drops to negligable values for high frequencies.

In Figure $4 \mathrm{~b}$, the transverse coupling impedance (reactance) is shown, as function of the frequency and of the particle speed, with similar considerations with respect to the longitudinal impedance.

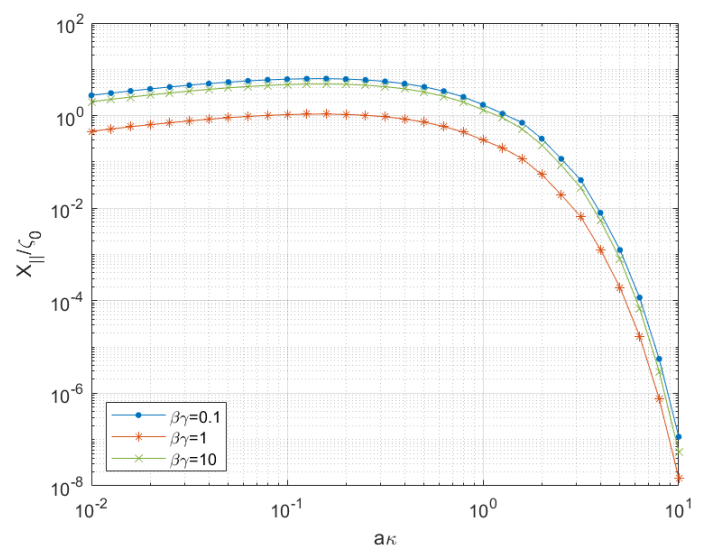

(a)

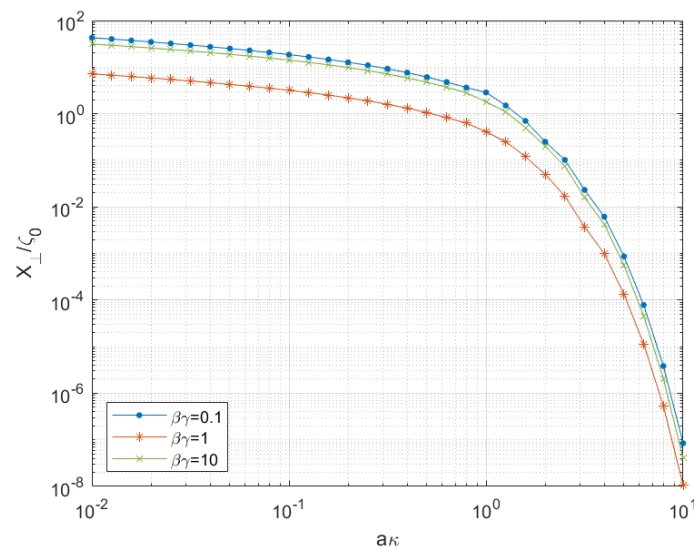

(b)

Figure 4. Normalized per unit length (a) longitudinal and (b) transverse coupling reactance for different frequencies $\left(r_{q}=0\right)$.

\section{Discussion}

A method for the evaluation of the coupling impedance of a particle travelling parallel close to a perfectly conducting angular slot has been presented. The method is accurate and effective, and can be easily generalized to similar geometries exhibiting angular variation in cylindrical coordinates.

As stated in the introduction, the angular slot is assumed to be perfectly conducting. This assumption is widely accepted in the literature on particle accelerator cavity design, for several reasons. First of all, the coupling impedance mainly takes into account the electromagnetic interaction between the particle and the surrounding structure, which is often mainly connected to structure shape. In this sense, the real part of the coupling impedance aims to take into account the diffractive losses. For this reason, most of the scientific paper consider the structure as perfectly conducting. In most of the cases where the conductivity of the structure is taken into account, this aspect is usually added to the perfectly conducting model with perturbative approaches and its effect usually smooths some rough behaviors but does not produce relevant changes. For this reason, the study of structures with perfectly conducting walls is often considered a valuable analysis, even if the inclusion of the finite conductivity is of course an added value. Regarding our specific problem, most of the analysis does not lose validity, even when adding the finite conductivity of the slot. Since the problem is formulated in the particle frame at first, the model is stationary regardless of whether the slot's conductivity is finite or not. So the first part of the paper is not affected. After applying the Lorentz transforms, once the current density is found in the strip frame, it is possible to evaluate the resistive power dissipation on the slot. Finally, in real particle accelerators, despite whether the particle speed is close to the light speed, the charge is extremely small and the current is not very high, usually hundreds of $\mathrm{mA}$ at most. Therefore, the current densities in practical cases are not huge.

The angular slot and the travelling charge are placed in an open space. In a particle accelerator, all the components are closed in a metallic pipe, which is necessary to maintain a vacuum. With respect to the proposed formulation, it is possible to add the presence of the pipe by partially changing the kernel of Equations (16) and (17). However, such a change just introduces some poles in the kernel, connected to the pipe resonances, independent of the asymmetry of the angular slot. Since the aim of 
the paper is to propose a method to deal with angularly asymmetric structures, just focussing on that and not mixing different phenomena, the external pipe has been neglected.

The proposed method has proven to be accurate and the series is quickly convergent. It is suitable to analyze structures with angular asymmetry. The obtained results can be used to benchmark numerical solutions or as reference geometry for more complex structures typical of particle accelerators.

Author Contributions: Conceptualization, D.A. and L.V.; methodology, D.A. and L.V.; software, D.A.; validation, D.A. and L.V.

Funding: This research received no external funding.

Conflicts of Interest: The authors declare no conflict of interest.

\section{Appendix A. Computation of the Linear System}

For low values of the argument, the kernel of the integral of Equation (16) exhibits a logarithmic singularity, namely

$$
K_{0}(z) \cong-\log (z / 2)-\gamma_{0},
$$

with $\gamma_{0}$ being the Eulero-Mascheroni constant. For its efficient numerical computation, it is worth adopting the variational form

$$
A_{n m}=A_{n m}^{\log }+A_{n m}^{0}
$$

where

$$
\begin{aligned}
A_{n m}^{0}=\int_{0}^{\pi} \int_{0}^{\pi}\left[K_{0}(\right. & \left.2 a w\left|\sin \left(\frac{\varphi_{a}}{2}\left(\cos \psi_{0}-\cos \psi^{\prime}\right)\right)\right|\right)+ \\
& \left.+\log \left(a w\left|\sin \left(\frac{\varphi_{a}}{2}\left(\cos \psi_{0}-\cos \psi^{\prime}\right)\right)\right|\right)+\gamma_{0}\right] \cos \left(m \psi_{0}\right) \cos \left(n \psi^{\prime}\right) d \psi_{0} d \psi^{\prime}
\end{aligned}
$$

The new integral in Equation (A3) has no singularities and can be numerically computed with minimal effort. Regarding the logarithmic, by means of the relevant expansion

$$
\log |\sin (x / 2)|=-\sum_{p=1}^{\infty} \frac{\cos p x}{p}-\log 2,
$$

with some manipulations it can be easily found that

$$
\begin{aligned}
A_{n m}^{\log }=-\int_{0}^{\pi} \int_{0}^{\pi}\left[\log \left(a w\left|\sin \left(\frac{\varphi_{a}}{2}\left(\cos \psi_{0}-\cos \psi^{\prime}\right)\right)\right|\right)+\gamma_{0}\right] \cos \left(m \psi_{0}\right) \cos \left(n \psi^{\prime}\right) d \psi_{0} d \psi^{\prime}= \\
\quad= \begin{cases}-\pi^{2}\left[\log (a w / 2)+\gamma_{0}\right], & m=n=0 \\
\pi^{2} \sum_{p=1}^{\infty} \frac{J_{m}\left(p / \varphi_{a}\right) J_{n}\left(p / \varphi_{a}\right)}{p}, & m+n \text { odd } \\
0, & m+n \text { even } .\end{cases}
\end{aligned}
$$




\section{References}

1. Lee, S.Y. Accelerator Physics; World Scientific Publishing: Singapore, 2018.

2. Kheifets, S.A.; Zotter, B.W. Impedances and Wakes in High-Energy Particle Accelerators; World Scientific: Singapore, 1998.

3. Heifets, S.A.; Kheifets, S.A. Coupling impedance in modern accelerators. Rev. Mod. Phys. 1993, 63, 631-673. [CrossRef]

4. Palumbo, L.; Vaccaro, V.G.; Zobov, M. Wake Fields and Impedance. 1995. Available online: https:/ /arxiv. org/abs/physics/0309023 (accessed on 21 May 2019).

5. Chao, A.W.; Mess, K.H. Handbook of Accelerator Physics and Engineering; World Scientific: Singapore, 1998.

6. Panofsky, W.; Wenzel, W. Transverse deflection of charged particles in radiofrequency fields. Rev. Sci. Instrum. 1956, 27, 967-977. [CrossRef]

7. Niedermayer, U.; Eidam, L.; Boine-Frankenheim, O. Analytic modeling, simulation and interpretation of broadband beam coupling impedance bench measurements. Nucl. Instrum. Methods Phys. Res. Sect. A Accel. Spectrom. Detect. Assoc. Equip. 2015, 776, 129-143. [CrossRef]

8. Zannini, C. Electromagnetic Simulation of CERN Accelerator Components and Experimental Applications. 2013. Available online: http:/ /inspirehep.net/record/1296518/ (accessed on 21 May 2019).

9. Campelo, J.E.; Ghini, J.; Salvant, B.; Argyropoulos, T.; Esteban Müller, J.; Ghini, J.; Lasheen, A.; Quartullo, D.; Salvant, B.; Shaposhnikova, E.; et al. An Extended SPS Longitudinal Impedance Model. 2015. Available online: http:/ /inspirehep.net/record/1417277/ (accessed on 21 May 2019).

10. Fujita, K.; Kawaguchi, H. Time Domain Numerical Simulation Method based on EFIE and MFIE for Axis-Symmetric Structure Objects. In Proceedings of the Progress in Electromagnetic Research Symposium 2004, Pisa, Italy, 28-31 March 2004; pp. 627-630.

11. Kuehn, E. A mode-matching method for solving field problems in waveguide and resonator circuits. Arch. Fuer Elektron. Und Uebertragungstechnik 1973, 27, 511-518.

12. Legenkiy, M.N.; Butrym, A.Y. Method of mode matching in time domain. Prog. Electromagn. Res. 2010, 22, 257-283. [CrossRef]

13. Assante, D.; Davino, D.; Falco, S.; Schettino, F.; Verolino, L. Coupling impedance of a charge travelling in a drift tube. IEEE Trans. Mag. 2005, 41, 1924-1927. [CrossRef]

14. Assante, D.; Verolino, L. Efficient evaluation of the longitudinal coupling impedance of a plane strip. Prog. Electromagn. Res. 2012, 26, 251-265. [CrossRef]

15. Censor, D. Free-space relativistic low-frequency scattering by moving objects. Prog. Electromagn. Res. 2007, 72, 195-214. [CrossRef]

16. Censor, D. Application-oriented relativistic electrodynamics. Prog. Electromagn. Res. 2000, 29, 107-168. [CrossRef]

17. Idemen, M.; Alkumru, A. Influence of motion on the edge-diffraction. Prog. Electromagn. Res. 2008, 6, 153-168. [CrossRef]

18. Censor, D. Broadband spatiotemporal differential-operator representations for velocity depending scattering. Prog. Electromagn. Res. 2006, 58, 51-70. [CrossRef]

19. Sautbekov, S.S. Diffraction of plane wave by strip with arbitrary orientation of wave vector. Prog. Electromagn. Res. 2011, 21, 117-131. [CrossRef]

20. Handapangoda, C.C.; Premaratne, M.; Pathirana, P.N. Plane wave scattering by a spherical dielectric particle in motion: A relativistic extension of the mie theory. Prog. Electromagn. Res. 2011, 112, 349-379. [CrossRef]

21. Gradshteyn, I.S.; Ryzhik, I.M. Table of Integrals, Series, and Products, 7th ed.; Academic Press: New York, NY, USA, 2007.

22. Eswaran, K. On the solutions of a class of dual integral equations occurring in diffraction problems. Proc. $R$. Soc. Lond. 1990, A429, 399-427. [CrossRef]

23. Cesarano, C. Generalized Chebyshev polynomials. Hacet. J. Math. Stat. 2014, 43, 731-740.

24. Cesarano, C. Identities and generating functions on Chebyshev polynomials. Georgian Math. J. 2012, 19, 427-440. [CrossRef]

25. Cesarano, C. Integral representations and new generating functions of Chebyshev polynomials. Hacet. J. Math. Stat. 2015, 44, 541-552. [CrossRef] 
26. Cesarano, C.; Fornaro, F. A note on two-variable Chebyshev polynomials. Georgian Math. J. 2017, 24, 339-350. [CrossRef]

27. Assante, D.; Falco, S.; Lucido, M.; Panariello, G.; Schettino, F.; Verolino, L. Shielding effect of a strip of finite thickness. Electr. Eng. 2006, 89, 79-87. [CrossRef]

(C) 2019 by the authors. Licensee MDPI, Basel, Switzerland. This article is an open access article distributed under the terms and conditions of the Creative Commons Attribution (CC BY) license (http:/ / creativecommons.org/licenses/by/4.0/). 